\title{
Risk factors for mortality of 557 adult patients with COVID 19 in Babol, Northern Iran: a retrospective cohort study
}

\author{
Javanian $\mathrm{M}^{1}$, Bayani $\mathrm{M}^{1}$, Shokri $\mathrm{M}^{1}$, Sadeghi-Haddad-Zavareh $\mathrm{M}^{1}$, Babazadeh $\mathrm{A}^{1}$, Ghadimi $\mathrm{R}^{2}$, \\ Sepidarkish $\mathrm{M}^{3}$, Bijani $\mathrm{A}^{2}$, Yahyapour $\mathrm{Y}^{1}$, Barary $\mathrm{M}^{4}$, Hasanpour $\mathrm{AH}^{4}$, Ebrahimpour $\mathrm{S}^{1}$ \\ Infectious Diseases and Tropical Medicine Research Center, Health Research Institute, Babol University \\ of Medical Sciences, Babol, Iran. drsoheil1503@yahoo.com
}

\begin{abstract}
BACKGROUND: This study was aimed to investigate the risk factors for mortality in patients with COVID-19. METHODS: For this retrospective cohort study, we included 121 deceased and 436 discharged cases with COVID-19 in Babol, Northern Iran. The cases were between March 1 to April 1, 2020.

RESULTS: Multivariate Poisson regression analysis revealed that older age (aRR: 1.03, 95\% Cl: 1.01,

$1.05, \mathrm{p}<0.001$ ), hospital length of stay (aRR: $0.94,95 \% \mathrm{Cl}: 0.90,0.97, \mathrm{p}=0.003)$, ICU admission (aRR:

4.34, 95\% Cl: 2.95, 6.37, p < 0.001), cerebrovascular disease (aRR: 1.96, 95\% Cl: 1.20, 3.19, p = 0.007), ventilator-associated pneumonia (VAP) (aRR: 2.09, 95\% Cl: 1.22, 3.55, p =0.006), septic shock (aRR: 2.98 , 95\% Cl: 1.44, 6.19, $\mathrm{p}=0.003$ ), acute respiratory distress syndrome (ARDS) (aRR: 3.80, 95\% Cl: 2.28, 6.31, $\mathrm{p}<0.001$ ), acute kidney failure (AKF) (aRR: $1.45,95 \% \mathrm{Cl}: 1.12,3.76, p=0.021$ ), acute heart failure (AHF) (aRR: $1.63,95 \% \mathrm{Cl}: 1.01,2.62, \mathrm{p}=0.043)$ and lymphocyte count (aRR: 3.01, 95\% Cl: 1.99, 4.57, p<0.001) were associated with mortality.

CONCLUSION: Findings showed that elderly with comorbidities such as cerebrovascular diseases had an increased risk of death. Some complications such as: pneumonia, septic shock, ARDS, AHF, and AKF played crucial roles as well death (Tab. 2, Ref. 25). Text in PDF www.elis.sk

KEY WORDS: COVID-19, mortality, clinical characteristics, laboratory findings.
\end{abstract}

\section{Introduction}

In late December 2019, local health authorities reported many cases of novel coronavirus (2019-nCoV)-infected pneumonia (NCIP) of unknown cause, which were possibility related to wet markets in Wuhan, a city in China's Hubei province $(1,2)$. The infection spread quickly across the globe. Subsequently, the 2019 novel coronavirus (COVID-19) was reported as a global health emergency by the end of January 2020 (3). In early January of 2020, COVID-19 was first isolated from some cases and confirmed as the cause of the NCIP. COVID-19 was classified in the betacoronavirus lineage $2 \mathrm{~b}$ (4). Several reports described that COVID-19 had about $80 \%$ homology with severe acute respiratory

${ }^{1}$ Infectious Diseases and Tropical Medicine Research Center, Health Research Institute, Babol University of Medical Sciences, Babol, Iran, ${ }^{2} \mathrm{So}-$ cial Determinants of Health Research Center, Health Research Institute, Babol University of Medical Sciences, Babol, Iran, ${ }^{3}$ Department of Biostatistics and Epidemiology, Babol University of Medical Sciences, Babol, Iran, and ${ }^{4}$ Student Research Committee, Babol University of Medical Sciences, Babol, Iran

Address for correspondence: S. Ebrahimpour, Infectious Diseases and Tropical Medicine Research Center, Health Research Institute, Babol University of Medical Sciences, Babol, Iran.

Phone: +989111149309, Fax: +981132207918

Acknowledgements: This work was supported by Babol University of Medical sciences Project, no. 724132930. syndrome-related coronavirus (SARS-CoV). Also, the homology between the COVID-19 genome and the bat SARS-like coronavirus genome is greater than $90 \%$ (5). Studies showed that the clinical features of COVID-19 may range from asymptomatic to multi-organ failure (e.g., acute respiratory distress syndrome (ARDS), shock, acute cardiac injury, acute kidney injury, and acute liver injury) and admission to the intensive care unit (ICU) and death (6). In the other words, most common clinical characteristics of COVID-19 included: fever, chills, non-productive cough, shortness of breath, muscle pain, and lymphopenia, along with some radiographic findings of pneumonia $(7,8)$. Mortality rates were reported in several studies. In the retrospective case series study, Chen et al reported 799 cases with COVID-19. This research showed that 113 patients died (overall mortality of up to $14 \%$ ) and 161 cases discharged [Chen, 2020 \#1](9). Despite several studies on COVID-19, clinical data from deceased cases did not appear to be sufficient. This may be due to the insufficient number of cases being examined. This study reported the clinical characteristics of COVID-19 patients admitted to 3 hospitals in Babol, Iran and factors associated with mortality.

\section{Methods}

Study design, ethical considerations, and participants

The current study protocol was approved by the Ethics Committee of Babol University of Medical Sciences, Babol, Iran (Code: IR. 
MUBABOL.HRI.REC.1398.349). Five hundred fifty-seven cases with confirmed COVID-19 admitted to 3 central hospitals affiliated to Babol University of Medical Sciences from March 1 to April 1, 2020, were enrolled. All cases with COVID-19 enrolled in the recent research were diagnosed according to WHO interim guidance for 2019 novel coronavirus (6th edition) (10). In other words, all patients with physician- and laboratory-confirmed (positive in nasopharyngeal/ throat swab specimens by reverse transcription polymerase chain reaction (RT-PCR)) COVID-19 infection were included, while suspected cases with similar clinical symptoms were excluded from this study. All recovered patients with $\mathrm{CO}-$ VID-19 had completely resolved signs and symptoms, had a significant improvement in pulmonary (blood oxygen saturation $(\mathrm{SpO} 2) \geq 93 \%$ ) and extrapulmonary organ dysfunction, and no longer needed a supportive care before hospital discharge. The requirement for an informed consent was waived because of the emergency conditions of this disease. These treatment centres cover a large population of people living in Mazandaran province, North of Iran. Over the course of the COVID-19 pandemic, 410 beds have been allocated across the board to treat COVID-19 patients and ICU capacity at our hospitals was increased to 60 beds. The clinical outcomes such as hospital discharge, length of stay, and death were followed up to April 20, 2020.

\section{Data Collection}

The patient's medical records were investigated by an experienced team of researchers and physicians of Infectious Diseases Research Center of Babol University of Medical Sciences. The epidemiological, clinical, laboratory, and radiological findings, medications and outcomes information were collected with a data collection checklist from the electronic medical records. Moreover, the recorded data of patients comprised demographic information, past medical history $(\mathrm{PMH})$, underlying medical conditions, signs, symptoms, para clinical data, and treatments (i.e., antivirals, antibiotics corticosteroids, breathing supports).

\section{Statistical analysis}

Continuous variables were described by the mean (SD) or median (IQR) as appropriate. Categorical variables were described
Tab. 1. Baseline characteristics of 557 patients hospitalized in 3 central hospitals affiliated to Babol University of Medical Sciences with COVID-19.

\begin{tabular}{|c|c|c|c|c|}
\hline & $\begin{array}{l}\text { All patients } \\
(\mathrm{n}=557)\end{array}$ & $\begin{array}{c}\text { Deceased patients } \\
(\mathrm{n}=121)\end{array}$ & $\begin{array}{c}\text { Survivor patients } \\
(\mathrm{n}=436)\end{array}$ & $\mathrm{p}^{*}$ \\
\hline Age, mean (SD), y & $60.2(15.3)$ & $67.9(13.9)$ & $58.0(15.0)$ & $<0.001$ \\
\hline \multicolumn{5}{|l|}{ Sex } \\
\hline Male & $305(54.7)$ & 75 (61.9) & $230(52.7)$ & \multirow{2}{*}{0.071} \\
\hline Female & $252(45.1)$ & $46(38.0)$ & $206(47.1)$ & \\
\hline \multicolumn{5}{|l|}{ Comorbidities } \\
\hline Hypertension & $148(26.5)$ & $44(36.3)$ & $104(23.8)$ & 0.006 \\
\hline Diabetes & $161(28.9)$ & $43(35.5)$ & $118(27.0)$ & 0.069 \\
\hline Malignancy & $27(4.8)$ & $10(8.2)$ & $17(3.8)$ & 0.048 \\
\hline Cardiovascular disease & $131(23.5)$ & $36(29.7)$ & $95(21.7)$ & 0.068 \\
\hline Chronic kidney disease & $21(3.7)$ & $5(4.1)$ & $16(3.6)$ & 0.813 \\
\hline Liver disease & $6(1.0)$ & $1(0.8)$ & $5(0.9)$ & 0.763 \\
\hline Cerebrovascular disease & $15(2.6)$ & $6(4.9)$ & $9(2.0)$ & 0.082 \\
\hline COPD & $18(3.2)$ & $5(4.1)$ & $13(2.9)$ & 0.527 \\
\hline \multicolumn{5}{|l|}{ Signs and symptoms } \\
\hline Fever & $360(64.6)$ & $69(57.0)$ & $291(66.7)$ & 0.048 \\
\hline Fatigue & $269(48.2)$ & $55(45.4)$ & $214(49.0)$ & 0.480 \\
\hline Dry cough & $353(63.3)$ & $68(56.2)$ & $285(65.3)$ & 0.064 \\
\hline Anorexia & $216(38.7)$ & $39(32.2)$ & $177(40.6)$ & 0.095 \\
\hline Myalgia & $109(19.5)$ & $17(14.0)$ & $92(21.1)$ & 0.084 \\
\hline Dyspnoea & $399(71.6)$ & $92(76.0)$ & $307(70.4)$ & 0.225 \\
\hline Expectoration & $142(25.4)$ & $20(16.5)$ & $122(27.9)$ & 0.011 \\
\hline Pharyngalgia & $68(1.2)$ & $7(5.7)$ & $61(13.9)$ & 0.015 \\
\hline Diarrhoea & $42(7.5)$ & $2(1.6)$ & $40(9.1)$ & 0.006 \\
\hline Constipation & $39(7.0)$ & $11(9.0)$ & $28(6.4)$ & 0.309 \\
\hline Haemoptysis & $10(1.7)$ & $0(0)$ & $10(2.2)$ & 0.093 \\
\hline Dizziness & $100(17.9)$ & $18(14.4)$ & $82(18.8)$ & 0.319 \\
\hline Headache & $128(22.9)$ & $15(12.4)$ & $113(25.9)$ & 0.002 \\
\hline Vomiting & $154(27.6)$ & $25(20.6)$ & $129(29.5)$ & 0.128 \\
\hline Abdominal pain & $66(11.8)$ & $10(8.2)$ & $56(12.8)$ & 0.168 \\
\hline Chest pain & $109(19.5)$ & $17(14.0)$ & $92(21.1)$ & 0.084 \\
\hline Anosmia & $36(6.4)$ & $3(2.48)$ & $33(7.5)$ & 0.044 \\
\hline $\begin{array}{l}\text { Hospital admission } \\
\text { median (IQR), d }\end{array}$ & $\begin{array}{c}6 \\
(10,4) \\
\end{array}$ & $\begin{array}{c}6 \\
(10,3) \\
\end{array}$ & $\begin{array}{c}6 \\
(10,4) \\
\end{array}$ & 0.819 \\
\hline $\begin{array}{l}\text { Heart rate, } \\
\text { median (IQR), bpm }\end{array}$ & $\begin{array}{c}80 \\
(88,80)\end{array}$ & $\begin{array}{c}83 \\
(100,80)\end{array}$ & $\begin{array}{c}80 \\
(100,80)\end{array}$ & $<0.001$ \\
\hline $\begin{array}{l}\text { Respiratory rate, } \\
\text { median (IQR) }\end{array}$ & $\begin{array}{c}20 \\
(21,20)\end{array}$ & $\begin{array}{c}20 \\
(22,19)\end{array}$ & $\begin{array}{c}20 \\
(22,19)\end{array}$ & 0.627 \\
\hline $\begin{array}{l}\text { Arterial pressure, } \\
\text { median (range), } \mathrm{mm} \mathrm{Hg}\end{array}$ & $\begin{array}{c}115.8 \\
(130.7,105.6) \\
\end{array}$ & $\begin{array}{c}113.1 \\
(130.8,100.8) \\
\end{array}$ & $\begin{array}{c}120.6 \\
(130.6,110.6) \\
\end{array}$ & 0.743 \\
\hline \multicolumn{5}{|l|}{ Complications } \\
\hline ARDS & $8(1.4)$ & $4(3.3)$ & $4(0.9)$ & 0.051 \\
\hline Acute cardiac injury & $31(5.5)$ & $24(19.8)$ & $7(1.6)$ & $<0.001$ \\
\hline Arrhythmia & $13(2.3)$ & $11(9.0)$ & $1(0.2)$ & $<0.001$ \\
\hline Ventilator-associated pneumonia & $15(2.6)$ & $5(4.1)$ & $10(2.2)$ & 0.269 \\
\hline White blood cell count, $\times 10^{9} / \mathrm{L}$ & $7.4 \pm(3.8)$ & $9.7 \pm(4.0)$ & $7.0 \pm(4.0)$ & $<0.001$ \\
\hline Lymphocyte count, $\times 10^{9} / \mathrm{L}$ & $2.1 \pm(1.0)$ & $1.3 \pm(0.9)$ & $2.3 \pm(1.0)$ & $<0.001$ \\
\hline C-reactive protein $(\mathrm{mg} / \mathrm{L})$ & $7.7 \pm(5.9)$ & $10.3 \pm(6.8)$ & $7.1 \pm(5.4)$ & $<0.001$ \\
\hline
\end{tabular}

by counts (percentages). Clinical features of the discharged and deceased patients were compared using the Chi-square test for categorical variables and Student's t-test for continuous variables. We estimated the adjusted risk ratios (aRRs) and $95 \%$ CIs for the association between the clinical features and mortality using the Poisson regression model. The results showed by the adjusted risk ratio (aRR) with $95 \%$ CIs. The following variables were included in the model: age, gender, hospital length of stay, ICU admission, co- 
morbidities, laboratory markers, vital signs (10). Statistical analyses were performed on Stata 16.0 (Stata Corp, College Station, TX, USA). All statistical tests were two-tailed at the significance level of $p<0.05$.

\section{Results}

A total of 557 adult patients (aged $\geq$ 16 years) with a confirmed COVID-19, were enrolled in the study, including 436 (78.28 \%, 95\% CI: 74.85, 81.70), who were discharged and $121(21.72 \%, 95 \%$ CI: $18.29,25.14)$, who died. Table 1 shows the baseline clinical features of the patients. All 557 enrolled patients showed a bilateral involvement of chest CT scans. The median age of the patients was 61 years (IQR: 71, $50.75)$, ranging from 16 years to 97 years, and $45.1 \%$ of patient were female. The median duration of hospital length of stay was 6 days (IQR: 10,4), ranging from one day to 34 days. As expected, the deceased patients were significantly older (MD: 9.91, 95\% CI: $6.83,13.01 ; \mathrm{p}<0.001)$ and were more likely to have underlying diseases, including hypertension (RR: $1.57,95 \%$ CI: 1.14 ,

2.17; $\mathrm{p}=0.005)$, malignancy (RR: $1.76,95 \% \mathrm{CI}: 1.05,2.97 ; \mathrm{p}=$ 0.047 ), cerebrovascular diseases (RR: $1.88,90 \% \mathrm{CI}: 1.10,3.22$; $\mathrm{p}=0.081$ ), and cardiovascular diseases (RR: $1.37,90 \% \mathrm{CI}: 1.03$, $1.82 ; \mathrm{p}=0.067)$. Of the total patients, $399(94.79 \%, 95 \% \mathrm{CI}$ : $92.94,96.63)$ had symptoms with dyspnoea $(71.63 \%)$ being the most common symptom, followed by fever (64.63\%), dry cough $(63.37 \%)$ and fatigue $(48.29 \%)$. While 44 (36.36\%, 95\% CI: $27.79,44.93)$ of deceased patients had deteriorated vital signs, only $22(5.04 \%, 95 \% \mathrm{CI}: 2.99,7.10)$ of the discharged patients had adverse vital signs $(\mathrm{p}<0.001)$. Among all patients, $134(24.05 \%$, $95 \%$ CI: 20.50, 27.60) were admitted with $\mathrm{SpO}_{2}$ less than $90 \%$. The heart rate of the deceased patients was significantly higher than values of those, who were discharged (MD: 6.63, 95\% CI: $3.49,9.17 ; \mathrm{p}<0.001)$. Also, the mean of $\mathrm{SpO}_{2}$ was significantly lower than the values of those, who were discharged (MD: -5.66 , $95 \% \mathrm{CI}:-7.82,-3.51 ; \mathrm{p}<0.001)$. Laboratory results showed that there were significant differences in the white blood cell counts (MD: $2.75,95 \%$ CI: $1.84,3.65 ; \mathrm{p}<0.001$ ), absolute values of lymphocytes (MD: $-1.01,95 \% \mathrm{CI}:-1.23,-0.76$; $\mathrm{p}<0.001$ ) and Creactive protein (CRP) (MD: 3.26, 95\% CI: 1.90, 4.62; $<<0.001$ ) between the two groups. Table 2 shows the association between clinical features of the patients and mortality of COVID-19 infection. For every day of hospital stay increase, the risk of death decreased by $6 \%$ (aRR: $0.94,95 \%$ CI: $0.90,0.97, p=0.003$ ). We found a strong positive association between ICU admission and COVID-19 mortality (aRR: 4.34, 95\% CI: 2.95, 6.37, p < 0.001). Patients with lymphopenia showed a significantly higher risk of death compared to normal lymphocyte count (aRR: $3.01,95 \% \mathrm{CI}$ :
$1.99,4.57, \mathrm{p}<0.001$ ). As the result, WBC counts (aRR: $1.29,95 \%$ CI: $0.85,1.94, p=0.224$ ) and CRP levels (aRR: $1.32,95 \%$ CI: 0.39 , $4.48, \mathrm{p}=0.651$ ) were ruled out as a risk factor for the COVID-19 mortality. Compared to the patients with appropriate vital signs, the risk of death was significantly higher in patients with ventilatorassociated pneumonia (VAP) (aRR: 2.09, 95\% CI: 1.22, 3.55, p = 0.006), septic shock (aRR: 2.98, 95\% CI: $1.44,6.19, \mathrm{p}=0.003$ ), ARDS (aRR: 3.80, 95\% CI: 2.28, 6.31, p < 0.001), acute heart failure (AHF) (aRR: 1.63, 95\% CI: 1.01, 2.62, p=0.043) and acute kidney failure (AKF) (aRR: 1.45, 95\% CI: 1.12, 3.76, $\mathrm{p}=0.021$ ). In addition, compared to the patients without comorbidities, the risk of COVID-19 mortality was also significantly higher among the patients with a history of cerebrovascular diseases (aRR: 1.96, $95 \%$ CI: $1.20,3.19, \mathrm{p}=0.007)$. The adjusted RR for other comorbidities in association with COVID-19 mortality was not estimated due to a sparse data and rare exposure. The association between gender and COVID-19 mortality was not significant (aRR: 1.09, $95 \%$ CI: $0.73,1.62, p=0.662$ ). Multivariable Poisson regression analysis revealed that older age (aRR: $1.03,95 \%$ CI: $1.01,1.05$, $\mathrm{p}<0.001$ ) was independently associated with hospital mortality. In our country, based on the standard treatment protocol, Kaletra (lopinavir/ ritonavir), hydroxychloroquine sulfate and chloroquine phosphate drugs were prescribed for COVID-19 patients. However, in some patients, medications such as interferons and antibiotics were also prescribed due to lack of appropriate response to treatment. Therefore, all the patients received antiviral treatment, including a single $400 \mathrm{mg}$ oral dose of hydroxychloroquine sulfate, or a single $500 \mathrm{mg}$ oral dose of chloroquine phosphate along 
with Kaletra two $200 \mathrm{mg}$ tablets BD, for 5-14 days. Furthermore, cephalosporins and glycopeptide antibiotics were also used for some patients with suspected staphylococcal infections.

\section{Discussion}

The current study described the main differences in clinical presentations between deceased patients with COVID-19 and those, who survived. The mean age of deceased cases was older than that of recovered cases. This study showed a direct association between ICU admission and mortality. Lymphopenia was more common in deceased patients than in recovered patients. The risk of death was higher in patients with VAP, septic shock, ARDS, AHF and AKF. Also, the risk of COVID-19 mortality was higher among cases with a history of cerebrovascular diseases. The results of such studies contribute to our knowledge of the factors affecting the mortality. As the result, it will speed up diagnostic and treatment processes of the patients and reduce mortality as well. The clinical spectrum of COVID-19 varies from asymptomatic forms to death (11). Here, the recent study including some clusters of patients showed a considerable mortality rate for COVID-19. This is partly due to a large proportion of critically ill cases admitted to our hospitals at this time. It is important to note, that the mortality rate of COVID-19 is lower than for SARS-CoV and MERS-CoV (12). Though, COVID-19 has verified more deadly for different reasons, such as commonly transition through direct person-to-person contacts and atypical symptoms at during early-stage infection $(13,14)$. Yang et al reported that the mortality rate in critically ill cases with COVID-19 was higher than that in critically ill patients with SARS-CoV (15). The overall mortality rate for confirmed COVID-19 patients was found to be higher in elderly $(16,17)$. In other words, elderly patients are at high risk of fatality with $\mathrm{CO}-$ VID-19. It was agreed with our results, that the age of deceased patients was higher than that of the survivors. There are several reasons for this issue such as: age-related decline in immune function and chronic comorbidities due to their weaker immune system (16). Moreover, based on the latest researches on clinical features of COVID-19 patients who required ICU admission, coexisting conditions, and elderly, are believed to be serious risk factors for death. As such, a high-quality monitoring seems necessary for the patients at risk. In our study, of the total cases, 399 had symptoms with dyspnoea $(71.63 \%)$ being the most common symptom, followed by fever $(64.63 \%)$, dry cough $(63.37$ $\%$ ) and fatigue (48.29\%). The incidence of symptoms including fever, expectoration, pharingalgia, diarrhoea, headache, and anosmia differed significantly between the non-survivors and recovered cases. These results were consistent with other researches, which showed common symptoms including: fever, fatigue, and dry cough (18). Moreover, recently there have been cases of gastrointestinal involvement such as diarrhoea and abdominal pain reported with COVID-19 (19). Also, the vital signs records showed that most deceased patients had tachycardia as well as lower $\mathrm{SpO}_{2}$ than the survivors. These signs and symptoms indicated that most deceased cases had been in a critical condition on admission. Therefore, clinicians should pay special attention to such symptoms, when dealing with this infection, because they can cause undesirable results with poor outcomes.

Some abnormalities of laboratory results between the deceased and recovered cases were considerable. Most of the dead cases developed severe lymphopenia. The presence of lymphopenia as a signature of severe COVID-19 was confirmed by many other studies. The researches suggested that the endothelial dysfunction induced endothelial cell death, blood-tissue barrier destruction, and leukocyte adhesion, which might describe the lymphopenia in severe COVID-19 (20). Moreover, other common laboratory abnormalities in deceased patients included leucocytosis and high CRP levels. However, by adjusting the various possible factors influencing these laboratory findings, we did not conclude that they are considered a risk factor for death. On the other hand, due to the small number of dead patients, this may be justified.

In the recent study, some complications such as: VAP, septic shock, ARDS, AHF, AKF, and comorbidities as cerebrovascular diseases were higher in deceased patients. Other studies showed that deceased cases might develop pulmonary and extrapulmonary organ dysfunctions, comprising like ARDS, acute kidney injury, sepsis, acute cardiac injury, shock, and acute liver injury in the later stages of COVID-19 (21). Among them, respiratory and neurological system complications along with the cardiac disorders were associated with a poor outcome (22). All of these warn physicians that particular attention should be paid to other complications, especially neurological and cardiac involvements, along with examining respiratory complications.

As yet, there are no drugs or vaccines approved to treat or prevent COVID-19 except some supportive therapies (23-25). In other words, for now the approach to COVID-19 include the control of possible sources of infection, decrease transmission probability between people, timely diagnosis, and treatments. Also, treatment with antibacterial agents seemed to be ineffective. In the present study, like many other studies, VAP has been suggested as an important factor in patient mortality. Therefore, in order to treat this infection and prevent the spread of antibiotic resistance, it is better to prepare appropriate guidelines on empirical antibiotic use in COVID-19 cases. The current study had some limitations. The relatively small sample size especially for nonsurvivors made it difficult to draw accurate conclusions. Thus, it is advisable to use bigger sample size in consequent researches to more accurately measure the association between several variables. Secondly, due to the retrospective study design, all laboratory tests were not estimated in all cases. It is primarily limited by being a retrospective analysis of laboratory data. Multiple clinicians were responsible for contributing to the administrative record, and the data were not validated by any central authority. In contrast, this study also had several strengths .It is a cohort study, so the first condition of causality, i.e. temporality, was realized. Also, in this study, detailed important variables for assessing the causal association among clinical features and in hospital mortality were gathered. Finally, we confront the confounding phenomenon by multivariate modified Poisson regression, and we estimated the risk ratio directly. 


\section{Conclusions}

Our data revealed that older patients with comorbidities such as cerebrovascular diseases had an increased risk of death with COVID-19. Some complications such as pneumonia, septic shock, ARDS, AHF, and AKF were associated with in-hospital mortality. It is also important to note that lymphopenia can be a signature of severe COVID-19 especially in deceased patients.

\section{References}

1. Rodriguez-Morales AJ, Cardona-Ospina JA, Gutiérrez-Ocampo E et al. Clinical, laboratory and imaging features of COVID-19: A systematic review and meta-analysis. Travel Med Infect Dis 2020: 101623.

2. Zhu N, Zhang $\mathbf{D}$, Wang $\mathbf{W}$ et al. A novel coronavirus from patients with pneumonia in China, 2019. N Engl J Med 2020 20; 382 (8): 727-733.

3. Yuen K-S, Ye Z-W, Fung S-Y, Chan C-P, Jin D-Y. SARS-CoV-2 and COVID-19: The most important research questions. Cell \& bioscience 2020; 10 (1): 1-5.

4. Helmy YA, Fawzy M, Elaswad A, Sobieh A, Kenney SP, Shehata AA. The COVID-19 Pandemic: A Comprehensive Review of Taxonomy, Genetics, Epidemiology, Diagnosis, Treatment, and Control. J Clin Med 2020; 9 4): 1225 .

5. Yang P, Wang X. COVID-19: a new challenge for human beings. Cell Mol Immunol 2020; 17 (5): 555-557.

6. Berlin DA, Gulick RM, Martinez FJ. Severe Covid-19. N Engl J Med 2020; 382 (19): 1787-1799

7. Javanian M, Masrour-roudsari J, Bayani M, Ebrahimpour S. Coronavirus disease 2019 (COVID-19): What we need to know. Caspian J Intern Med 2020; 11 (2): 235-236.

8. Mohseni Afshar Z, Ebrahimpour S, Javanian M, Vasigala VR, Masrour-roudsari J, Babazadeh A. Vital role of chest CT in diagnosis of coronavirus disease 2019 (COVID-19). Caspian J Intern Med 2020; 11 (3): 244-249.

9. Chen T, Wu D, Chen $\mathbf{H}$ et al. Clinical characteristics of 113 deceased patients with coronavirus disease 2019: retrospective study. Bmj 2020; 368.

10. Xu X-W, Wu X-X, Jiang X-G et al. Clinical findings in a group of patients infected with the 2019 novel coronavirus (SARS-Cov-2) outside of Wuhan, China: retrospective case series. BMJ 2020; 368.

11. Singhal T. A review of coronavirus disease-2019 (COVID-19). Indian J Pediatr 2020: 1-6.
12. Petrosillo N, Viceconte G, Ergonul O, Ippolito G, Petersen E. COVID-19, SARS and MERS: are they closely related? Clin Microbiol Infect 2020 .

13. Shen Y, Xu W, Li C et al. A Cluster of COVID-19 Infections Indicating Person-To-Person Transmission among Casual Contacts from Social Gatherings: An Outbreak Case-Contact Investigation. Available at SSRN 3563064. 2020.

14. Hu Z, Song C, Xu C et al. Clinical characteristics of 24 asymptomatic infections with COVID-19 screened among close contacts in Nanjing, China. Sci China Life Sci 2020; 63.

15. Yang X, Yu Y, Xu J et al. Clinical course and outcomes of critically ill patients with SARS-CoV-2 pneumonia in Wuhan, China: a single-centered, retrospective, observational study. The Lancet Resp Med 2020.

16. Liu K, Chen Y, Lin R, Han K. Clinical features of COVID-19 in elderly patients: A comparison with young and middle-aged patients. J Infect 2020; 80 (6): e14-e18.

17. Guan W-j, Ni Z-y, Hu Y et al. Clinical Characteristics of Coronavirus Disease 2019 in China. N Engl J Med 2020; 382 (18): 1708-1720.

18. Harapan H, Itoh N, Yufika A et al. Coronavirus disease 2019 (COVID-19): A literature review. J Infect Pub Health 2020.

19. Ollarves-Carrero MF, Rodriguez-Morales AG, Bonilla-Aldana DK, Rodriguez-Morales AJ. Anosmia in a healthcare worker with COVID-19 in Madrid, Spain. Travel Med Infect Dis 2020:101666.

20. Bermejo-Martin JF, Almansa R, Menéndez R, Mendez R, Kelvin DJ, Torres A. Lymphopenic community acquired pneumonia as signature of severe COVID-19 infection. J Infect 2020.

21. Martins-Filho PR, Tavares CSS, Santos VS. Factors associated with mortality in patients with COVID-19. A quantitative evidence synthesis of clinical and laboratory data. Eur J Intern Med 2020:S0953-6205 (20)30165-5.

22. Zaim S, Chong JH, Sankaranarayanan V, Harky A. COVID-19 and Multi-Organ Response. Curr Probl Cardiol 2020:100618.

23. Liu C, Zhou Q, Li Y, Garner LV et al. Research and Development on Therapeutic Agents and Vaccines for COVID-19 and Related Human Coronavirus Diseases. ACS Cent Sci 2020; 6 (3): 315-331.

24. Tobaiqy M, Qashqary M, Al-Dahery S et al. Therapeutic Management of COVID-19 Patients: A systematic review. Infect Prevent Pract 2020:100061.

25. Sanders JM, Monogue ML, Jodlowski TZ, Cutrell JB. Pharmacologic treatments for coronavirus disease 2019 (COVID-19): a review. JAMA 2020. 